\title{
Sampling parts of random integer partitions: a probabilistic and asymptotic analysis
}

\author{
LJuben Mutafchiev \\ American University in Bulgaria \\ 2700 Blagoevgrad, Bulgaria, and \\ Institute of Mathematics and Informatics \\ Bulgarian Academy of Sciences \\ email: ljuben@aubg.bg
}

(Received: September 8, 2014)

\begin{abstract}
Let $\lambda$ be a partition of the positive integer $n$, selected uniformly at random among all such partitions. Corteel et al. (1999) proposed three different procedures of sampling parts of $\lambda$ at random. They obtained limiting distributions of the multiplicity $\mu_{n}=\mu_{n}(\lambda)$ of the randomly-chosen part as $n \rightarrow \infty$. The asymptotic behavior of the part size $\sigma_{n}=\sigma_{n}(\lambda)$, under these sampling conditions, was found by Fristedt (1993) and Mutafchiev (2014). All these results motivated us to study the relationship between the size and the multiplicity of a randomly-selected part of a random partition. We describe it obtaining the joint limiting distributions of $\left(\mu_{n}, \sigma_{n}\right)$, as $n \rightarrow \infty$, for all these three sampling procedures. It turns out that different sampling plans lead to different limiting distributions for $\left(\mu_{n}, \sigma_{n}\right)$. Our results generalize those obtained earlier and confirm the known expressions for the marginal limiting distributions of $\mu_{n}$ and $\sigma_{n}$.
\end{abstract}

Mathematics Subject Classification(2010). 05A17, 60C05, 60F05.

Keywords: integer partition, part size, random sampling, limiting distribution.

\section{Introduction}

Partitioning integers into summands (parts) is a subject of intensive research in combinatorics, number theory and statistical physics. If $n$ is a positive integer, then by a partition, $\lambda$, of $n$, we mean a representation

$$
\lambda: \quad n=\sum_{j=1}^{n} j m_{j},
$$

in which $m_{j}$, called multiplicities of parts $j, j=1,2, \ldots, n$, are non-negative integers. We use $\Lambda(n)$ to denote the set of all partitions of $n$ and let $p(n)=|\Lambda(n)|$. The number $p(n)$ is determined asymptotically by the famous partition formula of Hardy and Ramanujan [9]:

$$
p(n) \sim \frac{1}{4 n \sqrt{3}} \exp \left(\pi \sqrt{\frac{2 n}{3}}\right), \quad n \rightarrow \infty .
$$

DOI: $10.1515 /$ puma-2015-0007 
A precise asymptotic expansion for $p(n)$ was found later by Rademacher [14] (more details may be also found in [2]). For instance, Rademacher's result implies that

$$
\begin{aligned}
& p(n)=\frac{1}{4 n \sqrt{3}} \exp \left(\pi \sqrt{\frac{2 n}{3}}\right)-\frac{1}{4 \pi \sqrt{2} n^{3 / 2}} \exp \left(\pi \sqrt{\frac{2 n}{3}}\right) \\
& +O\left(\exp \left(\frac{\pi}{2} \sqrt{\frac{2 n}{3}}\right)\right), \quad n \rightarrow \infty .
\end{aligned}
$$

Further on, we assume that, for fixed integer $n \geq 1$, a partition $\lambda \in \Lambda(n)$ is selected uniformly at random (uar), i.e. with probability $1 / p(n)$. In this way, each numerical characteristic of $\lambda$ can be regarded as a random variable defined on the space $\Lambda(n)$.

Corteel et al. [3] proposed and studied three procedures of sampling parts of a random partition $\lambda \in \Lambda(n)$. Basic statistics of a randomly selected part are the part size and its multiplicity. Corteel et al. 3] focused on the multiplicity $\mu_{n, j}=\mu_{n, j}(\lambda)(j=1,2,3$,$) of the randomly-selected part and found$ limiting distributions for $\mu_{n, j}$, as $n \rightarrow \infty$, in these three cases of sampling (here the subscript $j$ specifies the concrete sampling procedure that is followed; the definitions of these three sampling procedures will be given in the next section). In the same way, let $\sigma_{n, j}=\sigma_{n, j}(\lambda)(j=1,2,3)$ be the size of the randomly-selected part. Limit theorems for $\sigma_{n, j}$ were obtained in [6] and [13]. All these results motivated us to study the relationship between the size and the multiplicity of a randomly-selected part of a random integer partition. We describe it obtaining the joint limiting distributions of $\mu_{n, j}$ and $\sigma_{n, j}(\mathrm{j}=1,2,3)$ as $n \rightarrow \infty$. Our results generalize those obtained earlier in [6, 3, 13] and confirm the known expressions for the marginal limiting distributions of $\mu_{n, j}$ and $\sigma_{n . j}$.

We organize our paper as follows. In Section 2 we describe the sampling procedures proposed by Corteel et al. [3. The main results of this paper are stated in Section 3. The method of proof is also briefly described there. Section 4 contains some auxiliary facts on generating functions and some asymptotics that we need further. We present the proofs of our limit theorems in Sections 50.7.

\section{Basic random variables and definitions of the sampling procedures}

For any $\lambda \in \Lambda(n)$ selected uar, we define the random variables

$$
\alpha_{j}^{(n)}=\alpha_{j}^{(n)}(\lambda)=\text { the number of parts of size } j \text { in } \lambda .
$$

By $I_{A}$ we denote the indicator of an event $A$ and, for any two real numbers $d, s \geq 1$ and integer $m \geq 1$, we set

$$
\begin{gathered}
Z_{d, s}^{(n)}=\sum_{1 \leq j \leq s} \alpha_{j}^{(n)} I_{\left\{\alpha_{j}^{(n)} \leq d\right\}}, \\
Y_{m, s}^{(n)}=\sum_{1 \leq j \leq s} I_{\left\{\alpha_{j}^{(n)}=m\right\}} .
\end{gathered}
$$

$\left(Z_{d, s}^{(n)}\right.$ counts the number of parts of size not grater than $s$ and multiplicity not greater than $d$ in a randomly-chosen partition $\lambda$, while $Y_{m, s}^{(n)}$ is the number of distinct parts with multiplicity $m$ and size 
not greater than $s$ ). Obviously,

$$
Z_{n}=\sum_{j=1}^{n} \alpha_{j}^{(n)}
$$

equals the total number of parts and

$$
Y_{n}=\sum_{j=1}^{n} I_{\left\{\alpha_{j}^{(n)}>0\right\}}
$$

- the number of distinct parts in $\lambda \in \Lambda(n)$.

To describe the sampling procedures introduced by Corteel et al. [3] we notice that they are twostep procedures that combine the outcomes of two experiments. Therefore, they lead to three different product probability spaces. Since in each procedure we first sample uar a partition $\lambda \in \Lambda(n)$, the probability space on $\Lambda(n)$, equipped with the uniform probability measure $\operatorname{Pr}(\lambda \in \Lambda(n))=1 / p(n)$, is included in each product space. The second steps of sampling are, however, different and therefore, for each different procedure we obtain a different product space and different product probability measure. In what follows next, we adopt the common notation $\mathbb{P}($.$) for the product probability measure of each$ sampling procedure and follow the concept of a product space developed in [8], Chapter 1.6. By $\mathbb{E}(X)$ we denote the expected value of the random variable $X$ defined on the integer partition space $\Lambda(n)$.

Procedure 1. Given a partition $\lambda \in \Lambda(n)$ chosen uar (step 1), we select a part uar among all $Z_{n}$ parts of $\lambda$ (without any bias, step 2). By the product measure formula [8], Chapter 1.6, (4) and (6)

$$
\begin{aligned}
& \mathbb{P}\left(\{\lambda \in \Lambda(n)\} \times\left\{\mu_{n, 1} \leq d, \sigma_{n, 1} \leq s\right\}\right) \\
& =\operatorname{Pr}(\lambda \in \Lambda(n)) \mathbb{P}\left(\mu_{n, 1} \leq d, \sigma_{n, 1} \leq s\right)=\left(\frac{1}{p(n)}\right)\left(\frac{Z_{d, s}^{(n)}}{Z_{n}}\right) .
\end{aligned}
$$

Summation over all $\lambda \in \Lambda(n)$ yields

$$
\mathbb{P}\left(\mu_{n, 1} \leq d, \sigma_{n, 1} \leq s\right)=\mathbb{E}\left(\frac{Z_{d, s}^{(n)}}{Z_{n}}\right) .
$$

Procedure 2. Given a partition $\lambda \in \Lambda(n)$ chosen uar (step 1), we select a part among all $Y_{n}$ different parts (step 2). Recalling definitions (5) and (7) of the random variables $Y_{m, s}^{(n)}$ and $Y_{n}$, respectively, we obtain in a similar way that

$$
\mathbb{P}\left(\{\lambda \in \Lambda(n)\} \times\left\{\mu_{n, 2}=m, \sigma_{n, 2} \leq s\right\}\right)=\left(\frac{1}{p(n)}\right)\left(\frac{Y_{m, s}^{(n)}}{Y_{n}}\right)
$$

and

$$
\mathbb{P}\left(\mu_{n, 2}=m, \sigma_{n, 2} \leq s\right)=\mathbb{E}\left(\frac{Y_{m, s}^{(n)}}{Y_{n}}\right) .
$$

Procedure 3. Given a partition $\lambda \in \Lambda(n)$ chosen uar (step 1), we select a part of $\lambda$ with the probability proportional to its size and multiplicity (step 2). Thus we set

$$
\mathbb{P}\left(\{\lambda \in \Lambda(n)\} \times\left\{\mu_{n, 3}=m, \sigma_{n, 3} \leq s\right\}\right)=\left(\frac{1}{p(n)}\right)\left(\frac{m}{n}\right) \sum_{1 \leq j \leq s} j I_{\left\{\alpha_{j}^{(n)}=m\right\}},
$$


which in turn implies that

$$
\mathbb{P}\left(\mu_{n, 3}=m, \sigma_{n, 3} \leq s\right)=\frac{m}{n} \sum_{1 \leq j \leq s} j \operatorname{Pr}\left(\alpha_{j}^{(n)}=m\right) .
$$

REMARK 2.1 Sampling procedure 3 can be interpreted in terms of Ferrers diagrams - the graphical representations of the integer partitions $\lambda \in \Lambda(n)$ [2]. It is obtained as follows. We use the notation $\lambda_{k}$ to denote the $k$ th largest part of $\lambda$ for $k$ a positive integer; if the number of parts $Z_{n}$ of $\lambda$ is $<k$, then $\lambda_{k}=0$. The Ferrers diagram illustrates (1) by a two-dimensional array of dots, composed by $\lambda_{1}$ dots in the first (most left) row, $\lambda_{2}$ dots in the second row, ..., $\lambda_{Z_{n}}$ dots in the last $Z_{n}$ th row. Therefore, a Ferrers diagram may be considered as a union of disjoint blocks (rectangles) of dots with base $j$ and height $\alpha_{j}^{(n)}$ (the multiplicity of part $j$ ). So, 10 and 11 imply that the sampling probability in Procedure 3 is proportional to the area of the block to which the chosen part belongs.

\section{Statement of the main results and brief description of the method of proof}

For sampling procedures 1 - 3, we have proved the following limit theorems.

THEOREM 3.1 For the reals $u$ and $v$, we let

$$
F(u, v)= \begin{cases}0 & \text { if } \min \{u, v\} \leq 0 \\ 0 & \text { if } \min \{u, v\}>0 \quad \text { but } u+v \leq 1 \\ u+v-1 & \text { if } 0<u \leq 1,0<v \leq 1 \quad \text { and } u+v>1 \\ \min \{1, v\} & \text { if } u>1 \text { and } 0<v \leq 1 \\ \min \{1, u\} & \text { if } v>1 \text { and } 0<u \leq 1 \\ 1 & \text { if } u>1 \text { and } v>1\end{cases}
$$

Then, we have

$$
\lim _{n \rightarrow \infty} \mathbb{P}\left(\frac{2 \log \mu_{n, 1}}{\log n} \leq u, \frac{2 \log \sigma_{n, 1}}{\log n} \leq v\right)=F(u, v) .
$$

Theorem 3.2 Let $0<t<\infty$. Then, for any positive integer $m$, we have

$$
\lim _{n \rightarrow \infty} \mathbb{P}\left(\mu_{n, 2}=m, \frac{\pi \sigma_{n, 2}}{\sqrt{6 n}} \leq t\right)=\int_{0}^{t} e^{-m y}\left(1-e^{-y}\right) d y .
$$

Theorem 3.3 Let $0<t<\infty$. Then, for any positive integer $m$, we have

$$
\lim _{n \rightarrow \infty} \mathbb{P}\left(\mu_{n, 3}=m, \frac{\pi \sigma_{n, 3}}{\sqrt{6 n}} \leq t\right)=\frac{6 m}{\pi^{2}} \int_{0}^{t} y\left(1-e^{-y}\right) e^{-m y} d y .
$$

REMARK 3.4 Since the inequalities $\frac{2 \log \mu_{n, 1}}{\log n} \leq u, \frac{2 \log \sigma_{n, 1}}{\log n} \leq v$ are equivalent to $\mu_{n, 1} \leq n^{u / 2}, \sigma_{n, 1} \leq n^{v / 2}$, respectively, Theorem 3.1 implies that the proportion of parts of size $\leq n^{v / 2}$ and multiplicity $\leq n^{u / 2}$, $0<u, v<1$, is approximately equal to $u+v-1$ if $u+v>1$; if $u+v \leq 1$ this proportion approaches zero as $n \rightarrow \infty$. For the other two sampling procedures, Theorems 3.2 and 3.3 show that typically chosen part sizes are of order const $\sqrt{n}$, while their multiplicities are finite - both converge weakly to discrete random variables whose support is the set $\{1,2, \ldots\}$. 
REMARK 3.5 For the sake of completeness, we present here a list of the known marginal limiting distributions for the size and multiplicity of the randomly-chosen part. They can be obtained as corollaries of Theorems $3.1+3.3$. Proper references are also given.

$$
\lim _{n \rightarrow \infty} \mathbb{P}\left(\frac{2 \log \mu_{n, 1}}{\log n} \leq t\right)=t, \quad 0<t<1
$$

[3], p. 195;

$$
\lim _{n \rightarrow \infty} \mathbb{P}\left(\frac{2 \log \sigma_{n, 1}}{\log n} \leq t\right)=t, \quad 0<t<1
$$

[6], p. 712 ;

$$
\lim _{n \rightarrow \infty} \mathbb{P}\left(\mu_{n, 2}=m\right)=\frac{1}{m(m+1)}, \quad m=1,2, . .
$$

[3], p. 192;

$$
\lim _{n \rightarrow \infty} \mathbb{P}\left(\frac{\pi \sigma_{n, 2}}{\sqrt{6 n}} \leq t\right)=1-e^{-t} \quad 0<t<\infty
$$

[13], Theorem 2;

$$
\lim _{n \rightarrow \infty} \mathbb{P}\left(\mu_{n, 2}=m\right)=\frac{6(2 m+1)}{\pi^{2} m(m+1)^{2}}, \quad m=1,2, \ldots
$$

[3], p. 195;

$$
\lim _{n \rightarrow \infty} \mathbb{P}\left(\frac{\pi \sigma_{n, 3}}{\sqrt{6 n}} \leq t\right)=\frac{6}{\pi^{2}} \int_{0}^{t} \frac{y}{e^{y}-1} d y, \quad 0<t<\infty
$$

[13], Theorem 3.

We conclude this section with a description of our method of proof. It combines probabilistic with analytical tools. We employ Fristedt's conditioning device [6], which allows to transfer probability distributions of linear combinations of the multiplicities $\alpha_{j}^{(n)}$ into conditional distributions of the corresponding linear combinations of independent and geometrically distributed random variables. Using this method, we show that, as $n \rightarrow \infty$, the expected values in (8) and $(9)$ are close to the ratios of the expectations of the random variables that are involved there. The asymptotic behavior of the expectations of $Y_{n}$ and $Z_{n}$, defined by (7) and (6), respectively, is well known:

$$
\begin{gathered}
\mathbb{E}\left(Y_{n}\right) \sim \sqrt{6 n} / \pi, \\
\mathbb{E}\left(Z_{n}\right) \sim(\sqrt{6 n} / 2 \pi) \log n
\end{gathered}
$$

(see [16] and [3], respectively). We use combinatorial enumeration identities for generating functions, Cauchy coefficient formula and the saddle-point method in terms of Hayman admissibility theory [10] (see also [5], Chapter VIII.5) to obtain the asymptotic behavior of $\mathbb{E}\left(Z_{d, s}^{(n)}\right)$ (see (8)). Finally, (9) ) and (11) are analyzed using an approach developed by Corteel et al. [3] and based on Euler-MacLaurin sum formula. 


\section{Generating functions and the analytical background of the proofs}

We start with the notation $g(x)$ for the generating function of the sequence $\{p(n)\}_{n \geq 1}$. For $|x|<1$, $g(x)$ admits the well known representation

$$
g(x)=1+\sum_{n=1}^{\infty} p(n) x^{n}=\prod_{k=1}^{\infty}\left(1-x^{k}\right)^{-1}
$$

(see e.g. [2], Theorem 1.1). Our first lemma is related to the probability generating function and the expectation of the random variable $Z_{d, s}^{(n)}$, defined by 4 .

Lemma 4.1 For any reals $d, s \geq 1$ and complex variables $x$ and $z$, satisfying $|x|<1$ and $|z|<1$, we have

$$
1+\sum_{n=1}^{\infty} p(n) x^{n} \mathbb{E}\left(z^{Z_{d, s}^{(n)}}\right)=g(x) \prod_{1 \leq j \leq s} \frac{\left(1-\left(z x^{j}\right)^{d+1}\right)\left(1-x^{j}\right)}{1-z x^{j}} .
$$

Moreover

$$
\begin{aligned}
& 1+\sum_{n=1}^{\infty} p(n) x^{n} \mathbb{E}\left(Z_{d, s}^{(n)}\right) \\
& =g(x)\left(\sum_{1 \leq j \leq s} \frac{x^{j}}{1-x^{j}}-(d+1) \sum_{1 \leq j \leq s} \frac{x^{j(d+1)}}{1-x^{j(d+1)}}\right) \prod_{1 \leq j \leq s}\left(1-x^{j(d+1)}\right) .
\end{aligned}
$$

Proof. The generating function identity (15) follows from a more general argument developed in [15], Chapter V.5. To state it we need some preliminary notations. We let $B \subset\{1,2, \ldots\}$ and let $\Omega_{j} \subset \mathcal{N}_{0}=\{0,1, \ldots\}, j \geq 1$, be a sequence of sets. By $\widetilde{\sum}$ we denote a sum over all $j \in B$, satisfying (1) with $m_{j} \in \Omega_{j}, j \geq 1$. Then, we have

$$
\prod_{j \in B} \sum_{m_{j} \in \Omega_{j}}\left(z_{j} x^{j}\right)^{m_{j}}=1+\sum_{n \geq 1} x^{n} \widetilde{\sum} z_{1}^{m_{1}} z_{2}^{m_{2}} \ldots z_{n}^{m_{n}},
$$

where $x, z_{1}, z_{2}, \ldots$ are formal variables. In 17 we set $B=\{1,2, \ldots,[s]\}$,

$$
\Omega_{j}=\left\{\begin{array}{lll}
\{0,1, \ldots,[d]\} & \text { if } & j \leq s, \\
\mathcal{N}_{0} & \text { if } & j>s
\end{array}\right.
$$

and

$$
x_{j}=\left\{\begin{array}{lll}
x & \text { if } & j \leq s \\
1 & \text { if } & j>s .
\end{array}\right.
$$

(Here $[s]$ and $[d]$ denote the integer parts of $s$ and $d$, respectively.) The required identity (15) now follows from (4) and (14). A differentiation with respect to $z$ in (15) leads to the the expectations of $Z_{d, s}^{(n)}$ and identity 16.

The next lemma establishes a similar generating function identity for the random variable $Y_{m, s}^{(n)}$ defined by (5). It can be proved repeating the argument from [3], Theorem 1. 
Lemma 4.2 For any real number $s \geq 1$, positive integer $m$ and complex variables $x$ and $z$, satisfying $|x|<1$ and $|z|<1$, we have

$$
1+\sum_{n \geq 1, j \geq 0} x^{n} \mathbb{E}\left(z^{Y_{m, s}^{(n)}}\right)=g(x) \prod_{1 \leq k \leq s}\left(1+(z-1) x^{m k}\left(1-x^{k}\right)\right) .
$$

This in turn implies that

$$
\mathbb{E}\left(Y_{m, s}^{(n)}\right)=\sum_{1 \leq k \leq s}(p(n-m k)-p(n-(m+1) k)) .
$$

Further on, for the sake of simplicity, we let

$$
c=\frac{\pi}{\sqrt{6}} .
$$

We notice that Hardy-Ramanujan-Rademacher's formula in its form (3) implies that

$$
p(n)=\frac{e^{2 c n^{1 / 2}}}{4 \sqrt{3} n}\left(1+O\left(n^{-1 / 2}\right)\right), \quad n \rightarrow \infty .
$$

Using this expression, Corteel et al. [3] have obtained the following asymptotic estimates.

Lemma 4.3 For enough large n, we have

$$
\begin{gathered}
\frac{p(n-m j)}{p(n)}=\left(1+O\left(\frac{m j}{n^{3 / 2}}\right)+O\left((n-m j)^{-1 / 2}\right)\right) e^{-c m j / n^{1 / 2}} \\
=\left\{\begin{array}{lll}
\left(1+O\left(n^{-1 / 2}\right)\right) e^{-c m j / n^{1 / 2}} & \text { if } & m j \leq n / 2, \\
O\left(e^{-c n^{1 / 2} / 2}\right) & \text { if } & m j>n / 2 .
\end{array}\right.
\end{gathered}
$$

Lemma 3 enables us to interpret the sum in $(18)$ as a Riemann integral sum.

Our next preliminary fact is related to Hardy-Ramanujan formula (2). We shall present it into a slightly different form, which will be used further to find the asymptotic of $\mathbb{E}\left(Z_{d, s}^{(n)}\right)$. To introduce the reader into the subject, we notice that Hardy-Ramanujan formula has been subsequently generalized in various directions most notably by Meinardus [11] (see also [2], Chapter 6). Meinardus obtained the asymptotic of the Taylor coefficients of infinite products of the form

$$
\prod_{k=1}^{\infty}\left(1-x^{k}\right)^{-b_{k}}
$$

under certain general assumptions on the sequence of non-negative numbers $\left\{b_{k}\right\}_{k \geq 1}$. Meinardus approach is based on considering the Dirichlet generating series

$$
D(z)=\sum_{k=1}^{\infty} b_{k} k^{-z}, \quad z=u+i v .
$$


Since we shall use this result, below we briefly describe Meinardus assumptions avoiding their precise statements as well as some extra notations and concepts. The first Meinardus assumption $\left(M_{1}\right)$ specifies the domain $\mathcal{H}=\left\{z: \Re(z)=u \geq-C_{0}\right\}, 0<C_{0}<1$, in the complex plane, in which $D(z)$ has an analytic continuation. The second one $\left(M_{2}\right)$ is related to the asymptotic behavior of $D(z)$, whenever $|\Im(z)|=|v| \rightarrow \infty$. A function of the complex variable $z$ which is bounded by $O\left(|\Im(z)|^{C_{1}}\right), 0<C_{1}<\infty$, in certain domain of the complex plane is called function of finite order. Meinardus second condition $\left(M_{2}\right)$ requires that $D(z)$ is of finite order in the whole domain $\mathcal{H}$. Finally, the Meinardus third condition $\left(M_{3}\right)$ implies a bound on the ordinary generating function of the sequence $\left\{b_{k}\right\}_{k \geq 1}$. It can be stated in a way simpler than the Meinardus original expression by the inequality

$$
\sum_{k=1}^{\infty} b_{k} e^{-k \omega} \sin ^{2}(\pi k u) \geq C_{2} \omega^{-\epsilon_{1}}, \quad 0<\frac{\omega}{2 \pi}<|u|<\frac{1}{2},
$$

for sufficiently small $\omega$ and some constants $C_{2}, \epsilon_{1}>0\left(C_{2}=C_{2}\left(\epsilon_{1}\right)\right)$ (see [7], p. 310).

It is known that Euler partition generating function $g(x)$ (which is obviously of the form (21)) satisfies the Meinardus scheme of conditions $\left(M_{1}\right)-\left(M_{3}\right)$ (see e.g. [2], Theorem 6.3).

The proof of our Theorem 3.1 will be based on an asymptotic analysis of a Cauchy integral stemming from (16). We shall apply there the saddle-point method in the sense of Hayman [10] (see also [5], Chapter VIII.5). In [10] Hayman studied a wide class of power series satisfying a set of relatively mild conditions and established general formulas for the asymptotic order of their coefficients. In the proof of Theorem 3.1 we shall essentially use that the generating function $g(x)$ is admissible in the sense of Hayman. To present Hayman's idea and show how it can be applied, we need to introduce some auxiliary notations.

We consider here a function $G(x)=\sum_{n=1}^{\infty} G_{n} x^{n}$ that is analytic for $|x|<\rho, 0<\rho<\infty$. For $0<r<\rho$, we let

$$
\begin{gathered}
a(r)=r \frac{G^{\prime}(r)}{G(r)}, \\
b(r)=r \frac{G^{\prime}(r)}{G(r)}+r^{2} \frac{G^{\prime \prime}(r)}{G(r)}-r^{2}\left(\frac{G^{\prime}(r)}{G(r)}\right) .
\end{gathered}
$$

In the statement of Hayman's result we use the terminology given in [5], Chapter VIII.5. We assume that $G(x)>0$ for $x \in\left(R_{0}, \rho\right) \subset(0, \rho)$ and satisfies the following three conditions.

Capture condition. $\lim _{r \rightarrow \rho} a(r)=\infty$ and $\lim _{r \rightarrow \rho} b(r)=\infty$.

Locality condition. For some function $\delta=\delta(r)$ defined over $\left(R_{0}, \rho\right)$ and satisfying $0<\delta<\pi$, one has

$$
G\left(r e^{i \theta}\right) \sim G(r) e^{i \theta a(r)-\theta^{2} b(r) / 2}
$$

as $r \rightarrow \rho$, uniformly for $|\theta| \leq \delta(r)$.

Decay condition.

$$
G\left(r e^{i \theta}\right)=o\left(\frac{G(r)}{\sqrt{b(r)}}\right)
$$

as $r \rightarrow \rho$, uniformly for $\delta(r) \leq \theta<\pi$.

Hayman Theorem. Let $G(x)$ be Hayman admissible function and $r=r_{n}$ be the unique solution in the interval $\left(R_{0}, \rho\right)$ of the equation

$$
a(r)=n
$$


Then the Taylor coefficients of $G(x)$ satisfy, as $n \rightarrow \infty$,

$$
G_{n} \sim \frac{G\left(r_{n}\right)}{r_{n}^{n} \sqrt{2 \pi b\left(r_{n}\right)}}
$$

with $b\left(r_{n}\right)$ given by 24 .

The next lemma presents an alternative formula for the partition function $p(n)$.

LEMMA 4.4 If $r=r_{n}$ satisfies (25) for sufficiently large $n$, then

$$
p(n) \sim \frac{r_{n}^{n} g\left(r_{n}\right)}{\sqrt{2 \pi b\left(r_{n}\right)}}, \quad n \rightarrow \infty,
$$

where $a\left(r_{n}\right)$ and $b\left(r_{n}\right)$ are given by (23) and (24) with $G(x) \equiv g(x)$.

Proof. Since in (14) we have $b_{k}=1, k \geq 1$, the Dirichlet generating series $(22)$ is $D(z)=\zeta(z)$, where $\zeta$ denotes the Riemann zeta function. We set in 23 and 24$) r=r_{n}=e^{-h_{n}}, h_{n}>0$, where $h_{n}$ is the unique solution of the equation

$$
a\left(e^{-h_{n}}\right)=n .
$$

([27) is an obvious modification of [25).) Granovsky et al. [7] showed that the first two Meinardus conditions imply that the unique solution of 27 has the following asymptotic expansion:

$$
h_{n}=\sqrt{\zeta(2) / n}+\frac{\zeta(0)}{2 n}+O\left(n^{-1-\beta}\right)=\frac{\pi}{\sqrt{6 n}}-\frac{1}{4 n}+O\left(n^{-1-\beta}\right),
$$

where $\beta>0$ is fixed constant (here we have also used that $\zeta(0)=-1 / 2$; see [1]). We also notice that 24 and 28 impliy that

$$
b\left(e^{-h_{n}}\right)=2 \zeta(2) h_{n}^{-3}+O\left(h_{n}^{-2}\right) \sim \frac{\pi^{2}}{3} h_{n}^{-3} \sim \frac{2 \sqrt{6}}{\pi} n^{3 / 2}
$$

(see [12, Lemma 2.2, with $D(z)=\zeta(z)$ ). Hence, by 27) and 291), $a\left(e^{-h_{n}}\right) \rightarrow \infty$ and $b\left(e^{-h_{n}}\right) \rightarrow \infty$ as $n \rightarrow \infty$, that is, Hayman's "capture" condition is satisfied with $r=r_{n}=e^{-h_{n}}$. To show next that Hayman's "decay" condition is satisfied by $g(x)$ we set

$$
\delta_{n}=\frac{h_{n}^{4 / 3}}{\Omega(n)}=\frac{\pi^{4 / 3}}{(6 n)^{2 / 3} \Omega(n)}\left(1+O\left(\frac{1}{\sqrt{n}}\right)\right)
$$

with $h_{n}$ given by (28), where $\Omega(n) \rightarrow \infty$ as $n \rightarrow \infty$ arbitrarily slowly. We can apply now an estimate for $\left|g\left(e^{-h_{n}+i \theta}\right)\right|$ established in a general form in [12, Lemma 2.4, using all three Meinardus conditions. It states that there are two positive constants $c_{0}$ and $\epsilon_{0}$, such that, for sufficiently large $n$,

$$
\left|g\left(e^{-h_{n}+i \theta}\right)\right| \leq g\left(e^{-h_{n}}\right) e^{-c_{0} h_{n}^{-\epsilon_{0}}}
$$

uniformly for $\delta_{n} \leq|\theta|<\pi$.

This, in combination with 29 , implies that $\left|g\left(e^{-h_{n}+i \theta}\right)\right|=o\left(g\left(e^{-h_{n}}\right) / \sqrt{b\left(e^{-h_{n}}\right)}\right)$ uniformly in the same range for $\theta$, which is just Hayman's "decay" condition. Finally, by Lemma 2.3 of [12], established 
using Meinardus conditions $\left(M_{1}\right)$ and $\left(M_{2}\right)$, Hayman's "locality" condition is also satisfied by $g(x)$. In fact, this lemma implies in the particular case $D(z)=\zeta(z)$ that

$$
e^{-i \theta n} \frac{g\left(e^{-h_{n}+i \theta}\right)}{g\left(e^{-h_{n}}\right)}=e^{-\theta^{2} b\left(e^{-h_{n}}\right) / 2}\left(1+O\left(1 / \Omega^{3}(n)\right)\right.
$$

uniformly for $|\theta| \leq \delta_{n}$, where $b\left(e^{-h_{n}}\right)$ and $\delta_{n}$ are determined by 29$)$ and (30), respectively. Hence all conditions of Hayman's theorem hold and we can apply it with $G_{n}=p(n), G(x)=g(x), r_{n}=e^{-h_{n}}$ and $\rho=1$ to find that

$$
p(n) \sim \frac{e^{n h_{n}} g\left(e^{-h_{n}}\right)}{\sqrt{2 \pi b\left(e^{-h_{n}}\right)}}, \quad n \rightarrow \infty,
$$

which completes the proof.

REMARK 4.5 To show that formula $(33)$ yields $(2)$, one has to replace $\sqrt{28}$ ) and $(29)$ in the right hand side of (33). The asymptotic of $g\left(e^{-\overline{h_{n}}}\right)$ is determined by a general lemma due to Meinardus [11] (see also [2], Lemma 6.1). Since $\zeta(0)=-1 / 2$ and $\zeta^{\prime}(0)=-\frac{1}{2} \log (2 \pi)$ (see [1]), in the particular case of $g\left(e^{-h_{n}}\right)$ this lemma implies that

$$
\begin{aligned}
& g\left(e^{-h_{n}}\right)=\exp \left(\zeta(2) h_{n}^{-1}-\zeta(0) \log h_{n}+\zeta^{\prime}(0)+O\left(h_{n}^{c_{1}}\right)\right) \\
& =\exp \left(\frac{\pi^{2}}{6 h_{n}}+\frac{1}{2} \log h_{n}-\frac{1}{2} \log (2 \pi)+O\left(h_{n}^{c_{1}}\right)\right), \quad n \rightarrow \infty,
\end{aligned}
$$

where $0<c_{1}<1$. The rest of the computation leading to $(2)$ is based on simple algebraic manipulations and cancellations.

\section{$5 \quad$ Proof of Theorem 3.1}

We base our proof on the definition of Sampling Procedure 1 and eq. (8). We want to replace the expected value in its right-hand side by the ratio $\mathbb{E}\left(Z_{d, s}^{(n)}\right) / \mathbb{E}\left(Z_{n}\right)$. So, we notice first that Erdös and Lehner [4] proved that, in probability, the total number of parts $Z_{n}$ is asymptotic to $\mathbb{E}\left(Z_{n}\right)$ as $n \rightarrow \infty$. Hence, for any $\epsilon>0$, the probability of the event

$$
A_{n}=\left\{\lambda \in \Lambda(n):\left|\frac{Z_{n}}{\mathbb{E}\left(Z_{n}\right)}-1\right|>\epsilon\right\}
$$

tends to 0 as $n \rightarrow \infty$. Further, we rewrite $(8)$ in the following way:

$$
\mathbb{P}\left(\mu_{n, 1} \leq d, \sigma_{n, 1} \leq s\right)=\mathbb{E}\left(\frac{Z_{d, s}^{(n)}}{Z_{n}} I_{A_{n}^{c}}\right)+\mathbb{E}\left(\frac{Z_{d, s}^{(n)}}{Z_{n}} I_{A_{n}}\right) .
$$

For $\lambda \in A_{n}^{c}$ and $0<\epsilon<1$, we have $(1-\epsilon) \mathbb{E}\left(Z_{n}\right) \leq Z_{n} \leq(1+\epsilon) \mathbb{E}\left(Z_{n}\right)$ and therefore,

$$
\frac{\mathbb{E}\left(Z_{d, s}^{(n)}\right)}{(1+\epsilon) \mathbb{E}\left(Z_{n}\right)} \leq \mathbb{E}\left(\frac{Z_{d, s}^{(n)}}{Z_{n}} I_{A_{n}^{c}}\right) \leq \frac{\mathbb{E}\left(Z_{d, s}^{(n)}\right)}{(1-\epsilon) \mathbb{E}\left(Z_{n}\right)} .
$$


Since $Z_{d, s}^{(n)} \leq Z_{n}$, the second summand in 34 is not greater than $\mathbb{P}\left(A_{n}\right)$. Hence, combining 34 and (35), we obtain

$$
\mathbb{P}\left(\mu_{n, 1} \leq d, \sigma_{n, 1} \leq s\right)=(1+O(\epsilon)) \frac{\mathbb{E}\left(Z_{d, s}^{(n)}\right)}{\mathbb{E}\left(Z_{n}\right)}+\mathbb{P}\left(A_{n}\right) .
$$

Letting $n \rightarrow \infty$ and then $\epsilon \rightarrow 0$ and replacing $\mathbb{E}\left(Z_{n}\right)$ by the right-hand side of (13), uniformly for $d, s \geq 1$, we finally get

$$
\mathbb{P}\left(\mu_{n, 1} \leq d, \sigma_{n, 1} \leq s\right) \sim \frac{2 \pi \mathbb{E}\left(Z_{d, s}^{(n)}\right)}{\sqrt{6 n} \log n}=\frac{2 c \mathbb{E}\left(Z_{d, s}^{(n)}\right)}{\sqrt{n} \log n},
$$

where $c$ is the constant from 19 .

Our proof continues with an application of Cauchy coefficient formula to (16). We use the circle $x=e^{-h_{n}+i \theta},-\pi<\theta \leq \pi$, as a contour of integration and the notation

$$
\varphi_{d, s}(x)=\left(\sum_{1 \leq j \leq s} \frac{x^{j}}{1-x^{j}}-(d+1) \sum_{1 \leq j \leq s} \frac{x^{j(d+1)}}{1-x^{j(d+1)}}\right) \prod_{1 \leq j \leq s}\left(1-x^{j(d+1)}\right)
$$

to obtain

$$
p(n) \mathbb{E}\left(Z_{d, s}^{(n)}\right)=\frac{e^{n h_{n}}}{2 \pi} \int_{-\pi}^{\pi} g\left(e^{-h_{n}+i \theta}\right) \varphi_{d, s}\left(e^{-h_{n}+i \theta}\right) e^{-i \theta n} d \theta .
$$

Then, we break up the range of integration as follows:

$$
p(n) \mathbb{E}\left(Z_{d, s}^{(n)}\right)=J_{1}(d, s, n)+J_{2}(d, s, n),
$$

where

$$
\begin{gathered}
J_{1}(d, s, n)=\frac{e^{n h_{n}}}{2 \pi} \int_{-\delta_{n}}^{\delta_{n}} g\left(e^{-h_{n}+i \theta}\right) \varphi_{d, s}\left(e^{-h_{n}+i \theta}\right) d \theta, \\
J_{2}(d, s, n)=\frac{e^{n h_{n}}}{2 \pi} \int_{\delta_{n}<|\theta| \leq \pi} g\left(e^{-h_{n}+i \theta}\right) \varphi_{d, s}\left(e^{-h_{n}+i \theta}\right) d \theta
\end{gathered}
$$

and $\delta_{n}$ is defined by $(30)$.

In our next step we set

$$
d=n^{u / 2}, \quad s=n^{v / 2}, \quad 0 \leq u, v \leq 1
$$

and obtain estimates for the sums:

$$
\begin{gathered}
S_{1}=\sum_{1 \leq j \leq s} \frac{e^{-j h_{n}}}{1-e^{-j h_{n}}}, \\
S_{2}=\sum_{1 \leq j \leq s} \frac{e^{-j(d+1) h_{n}}}{1-e^{-j(d+1) h_{n}}} .
\end{gathered}
$$

Here the sequence $\left\{h_{n}\right\}_{n \geq 1}$ is defined by 28 . 
Using the approximation of a Riemann sum by an integral, 28, 41 and (19), for $S_{1}$ we get

$$
\begin{aligned}
& S_{1}=\left(1+O\left(\frac{1}{n}\right)\right) \sqrt{n} \sum_{1 \leq j \leq n^{v / 2}} \frac{e^{-c j / \sqrt{n}}}{1-e^{-c j / \sqrt{n}}} \frac{1}{\sqrt{n}} \\
& \sim \sqrt{n} \int_{1 / \sqrt{n}}^{n^{\frac{v-1}{2}}} \frac{e^{-c z}}{1-e^{-c z}} d z=\frac{\sqrt{n}}{c} \int_{c / \sqrt{n}}^{n^{\frac{v-1}{2}}} \frac{e^{-z}}{1-e^{-z}} d z \\
& =\frac{\sqrt{n}}{c} \log \left(\frac{1-e^{-c n^{\frac{v-1}{2}}}}{1-e^{-c / \sqrt{n}}}\right)=\frac{v \sqrt{n}}{2 c} \log n+O\left(n^{v / 2}\right) .
\end{aligned}
$$

In the same way one can show that

$$
S_{2}=\left\{\begin{array}{lll}
\frac{1-u}{2 c} n^{\frac{1-u}{2}} \log n+O\left(n^{\frac{1-u}{2}}\right) & \text { if } & v+u \geq 1 \\
\frac{v}{2 c} n^{\frac{1-u}{2}} \log n+O\left(n^{\frac{1-u}{2}}\right) & \text { if } & v+u<1
\end{array}\right.
$$

We are now ready to find an estimate for the second integral in $(38)$ (see $(40)$ ). First, we have

$$
\begin{aligned}
& \mid \prod_{1 \leq j \leq s}\left(1-e^{-h_{n} j(d+1)+i j \theta(d+1)} \mid\right. \\
& \leq \prod_{1 \leq j \leq s}\left(1-e^{-h_{n} j(d+1)}\right)+\prod_{1 \leq j \leq s} e^{-h_{n} j(d+1)}\left|1-e^{i j \theta(d+1)}\right| \\
& \leq 1+e^{-h_{n}(d+1) s}\left(1+\prod_{1 \leq j \leq s}\left|e^{i j \theta(d+1)}\right|\right)=1+2 e^{-h_{n}(d+1) s} \leq 3 .
\end{aligned}
$$

Hence, in terms of notations (37), 42) and (43), by (41), (44) and 445,

$$
\left|\varphi_{d, s}\left(e^{-h_{n}+i \theta}\right)\right|=O\left(\left(S_{1}+(d+1) S_{2}\right)=O(\sqrt{n} \log n), \quad-\pi \leq \theta \leq \pi .\right.
$$

Replacing this estimate and applying inequality 31 to the integrand of 40 , we obtain

$$
\left|J_{2}(d, s, n)\right|=O\left(e^{n h_{n}} g\left(e^{-h_{n}}\right) \sqrt{n}(\log n) e^{-c_{0} h_{n}^{-\epsilon_{0}}}\right) .
$$

The required estimate now follows from $(29)$ and $(33)$ in the following way:

$$
\begin{aligned}
& \left|J_{2}(d, s, n)\right|=O\left(\frac{e^{n h_{n}} g\left(-e^{-h_{n}}\right)}{\left.\sqrt{b\left(e^{-h_{n}}\right.}\right)} n^{1 / 2+3 / 4}(\log n) e^{-c_{0} h_{n}^{-\epsilon_{0}}}\right) \\
& =O\left(p(n) n^{5 / 4}(\log n) e^{-c_{0} h_{n}^{-\epsilon_{0}}}\right)=O\left(p(n) n^{5 / 4}(\log n) e^{-c_{0}^{\prime} n^{\epsilon_{0} / 2}}\right) \\
& =o(\sqrt{n}(\log n) p(n)),
\end{aligned}
$$

where $c_{0}^{\prime}>0$.

The estimate for $J_{1}(d, s, n)$ follows from Hayman's "locality" condition (32). First, we need to expand $\varphi_{d, s}$ by Taylor formula. We have

$$
\begin{aligned}
& \varphi_{d, s}\left(e^{-h_{n}+i \theta}\right)=\varphi_{d, s}\left(e^{-h_{n}}\right)+O\left(\left.|\theta| \frac{d}{d x} \varphi_{d, s}(x)\right|_{x=e^{-h_{n}}}\right) \\
& =\varphi_{d, s}\left(e^{-h_{n}}\right)+O\left(\left.\delta_{n} \frac{d}{d x} \varphi_{d, s}(x)\right|_{x=e^{-h_{n}}}\right) .
\end{aligned}
$$


To find the asymptotic of $\varphi_{d, s}\left(e^{-h_{n}}\right)$, in addition to 44 and $(45)$, we also need the limit of $\prod_{1 \leq j \leq s}(1-$ $e^{-j(d+1) h_{n}}$ ) as $n \rightarrow \infty$, whenever $d$ and $s$ satisfy (41) (see (37)). Using approximations by Riemann integrals as in the analysis of $S_{1}$ and $S_{2}$, it is easy to show that

$$
\prod_{1 \leq j \leq s}\left(1-e^{-j(d+1) h_{n}}\right)=\exp \left(\sum_{1 \leq j \leq s} \log \left(1-e^{-j(d+1) h_{n}}\right)\right) \rightarrow \begin{cases}1 & \text { if } \quad v+u \geq 1 \\ 0 & \text { if } \quad v+u<1\end{cases}
$$

Hence, from (41)-(45) it follows that

$$
\varphi_{d, s}\left(e^{-h_{n}}\right)\left\{\begin{array}{lll}
\sim \frac{u+v-1}{2 c} \sqrt{n} \log n & \text { if } & v+u \geq 1 \\
=o(\sqrt{n}) & \text { if } & v+u<1
\end{array}\right.
$$

The estimate of the error term in 48 is tedious and follows the same line of reasoning. We have

$$
\begin{aligned}
& \left.\frac{d}{d x} \varphi_{d, s}(x)\right|_{x=e^{-h_{n}}} \\
& =\left(\sum_{1 \leq j \leq s} \frac{j x^{j-1}}{\left(1-x^{j}\right)^{2}}-(d+1) \sum_{1 \leq j \leq s} \frac{j x^{j(d+1)}}{\left(1-x^{j(d+1)}\right)^{2}}\right) \\
& \times\left.\left(\prod_{1 \leq j \leq s}\left(1-x^{j(d+1)}\right)\right)\right|_{x=e^{-h_{n}}}+\left(\prod_{1 \leq j \leq s}\left(1-x^{j(d+1)}\right)\right) \\
& \times\left.\exp \left(-(d+1) \sum_{1 \leq j \leq s} \frac{j x^{j(d+1)-1}}{1-x^{j(d+1)}}\right)\right|_{x=e^{-h_{n}}} .
\end{aligned}
$$

It can be seen that the first two sums in the right-hand side of $(50)$ are of order $O(n \log n)$, while the first product factor is estimated by (46). Hence, the first summand in 50 is of order $O(n \log n)$. For the sum in the exponent of the second summand of the right-hand side of (50), one can show that there exists a constant $C>0$ such that

$$
\left.(d+1) \sum_{1 \leq j \leq s} \frac{j x^{j(d+1)-1}}{1-x^{j(d+1)}}\right|_{x=e^{-h_{n}}} \geq C \sqrt{n} \log n .
$$

Therefore the second summand in 50 is $O\left(e^{-C \sqrt{n} \log n)}\right)$. Hence

$$
\left.\frac{d}{d x} \varphi_{d, s}(x)\right|_{x=e^{-h_{n}}}=O(n \log n)
$$

and by 30 and 49 , the expansion in 48 becomes

$$
\varphi_{d, s}\left(e^{-h_{n}+i \theta}\right)=\varphi_{d, s}\left(e^{-h_{n}}\right)+O\left(\frac{n^{1 / 3} \log n}{\Omega(n)}\right)
$$


where $\Omega(n) \rightarrow \infty$ as $n \rightarrow \infty$ arbitrarily slowly. Inserting this estimate and (32) into (39) and applying the asymptotic for the partition function $p(n)$ from $(33)$, we obtain

$$
\begin{aligned}
& J_{1}(d, s, n)=\frac{e^{n h_{n}} g\left(e^{-h_{n}}\right)}{2 \pi}\left(\int_{-\delta_{n}}^{\delta_{n}} e^{-\theta^{2} b\left(e^{-d_{n}}\right) / 2}\left(1+O\left(1 / \Omega^{3}(n)\right) d \theta\right)\right. \\
& \times\left(\varphi_{d, s}\left(e^{-h_{n}}\right)+O\left(\frac{n^{1 / 3} \log n}{\Omega(n)}\right)\right) \\
& \sim \frac{e^{n h_{n}} g\left(e^{-h_{n}}\right)}{\sqrt{b\left(e^{-h_{n}}\right)} 2 \pi}\left(\int_{-\delta_{n} \sqrt{b\left(e^{-h_{n}}\right)}}^{\delta_{n} \sqrt{b\left(e^{-h_{n}}\right)}} e^{-y^{2} / 2} d y\right)\left(\varphi_{d, s}\left(e^{-h_{n}}\right)+O\left(\frac{n^{1 / 3} \log n}{\Omega(n)}\right)\right) \\
& \sim \frac{e^{n h_{n}} g\left(e^{-h_{n}}\right)}{\sqrt{b\left(e^{-h_{n}}\right)} 2 \pi}\left(\int_{-\infty}^{\infty} e^{-y^{2} / 2} d y\right)\left(\varphi_{d, s}\left(e^{-h_{n}}\right)+O\left(\frac{n^{1 / 3} \log n}{\Omega(n)}\right)\right) \\
& =\frac{e^{n h_{n}} g\left(e^{-h_{n}}\right)}{\sqrt{2 \pi b\left(e^{-h_{n}}\right)}}\left(\varphi_{d, s}\left(e^{-h_{n}}\right)+O\left(\frac{n^{1 / 3} \log n}{\Omega(n)}\right)\right) \\
& \sim p(n)\left(\varphi_{d, s}\left(e^{-h_{n}}\right)+O\left(\frac{n^{1 / 3} \log n}{\Omega(n)}\right)\right),
\end{aligned}
$$

where for the second asymptotic equivalence we have used $(29)$ and $(30)$ in order to get

$$
\delta_{n} \sqrt{b\left(e^{-h_{n}}\right)} \sim \frac{\pi^{5 / 6} \sqrt{2}}{6^{1 / 6} \Omega(n)} n^{1 / 12} \rightarrow \infty
$$

if $\Omega(n) \rightarrow \infty$ as $n \rightarrow \infty$ not too fast, so that $\frac{n^{1 / 12}}{\Omega(n)} \rightarrow \infty$. It is now clear that $38--40, \sqrt{47}$ and 52 yield

$$
p(n) \mathbb{E}\left(Z_{d, s}^{(n)}\right)=p(n) \varphi_{d, s}\left(e^{-h_{n}}\right)+o(p(n) \sqrt{n} \log n)
$$

and therefore

$$
\mathbb{E}\left(Z_{d, s}^{(n)}\right)=\varphi_{d, s}\left(e^{-h_{n}}\right)+o(\sqrt{n} \log n)
$$

The result of Theorem 3.1 now follows from (36), (13), (19) and (49).

\section{Proof of Theorem 3.2}

We base our proof on (9), Lemmas 2 and 3 and asymptotic equivalence 12 . To replace the expectation in the right hand side of 9 by the ratio $\mathbb{E}\left(Y_{m, s}^{(n)}\right) / \mathbb{E}\left(Y_{n}\right)$, similarly to what we did in the proof of Theorem 3.1, we shall study how unlikely is the event

$$
B_{n}=\left\{\lambda \in \Lambda(n):\left|\frac{c Y_{n}(\lambda)}{\sqrt{n}}-1\right|>\epsilon\right\}, \quad \epsilon>0,
$$

where $c$ is constant from (19). Using Fristeft's method [6], Corteel et al. [3] showed that

$$
\operatorname{Pr}\left(B_{n}\right) \leq e^{-c_{2} \sqrt{n}}, \quad c_{2}=c_{2}(\epsilon)>0 .
$$


REMARK 6.1 Fristedt's approach [6] is based on the identity

$$
\operatorname{Pr}\left(\alpha_{j}^{(n)}=m_{j}, j=1, \ldots, n\right)=\operatorname{Pr}\left(\gamma_{j}=m_{j}, j=1, \ldots, n \mid \sum_{j \geq 1} j \gamma_{j}=n\right),
$$

where $\left\{\gamma_{j}\right\}_{j \geq 1}$ is a sequence of independent geometrically distributed random variables, whose distribution is given by

$$
\operatorname{Pr}\left(\gamma_{j}=k\right)=\left(1-q^{j}\right) q^{j k}, \quad k=0,1, \ldots
$$

and $\left\{m_{j}\right\}_{j \geq 1}$ are non-negative integers. Eq. (54) holds for every fixed $q \in(0,1)$. It is natural to take $q$ so that $\operatorname{Pr}\left(\sum_{j \geq 1} j \gamma_{j}=n\right)$ is as large as possible. Fristedt's almost optimal choice for $q$ is $q=e^{-c / \sqrt{n}}$. Then, the bound in (53) is easily obtained using this value of $q$.

Next, we represent the probability in $(9)$ in the following way

$$
\mathbb{P}\left(\mu_{n, 2}=m, \sigma_{n, 2} \leq s\right)=\mathbb{E}\left(\frac{Y_{m, s}^{(n)}}{Y_{n}} I_{B_{n}^{c}}\right)+\mathbb{E}\left(\frac{Y_{m, s}^{(n)}}{Y_{n}} I_{B_{n}}\right),
$$

where $I_{B_{n}}$ and $I_{B_{n}^{c}}$ denote the indicators of events $B_{n}$ and $B_{n}^{c}$, respectively. Since, for any $\lambda \in B_{n}^{c}$,

$$
\frac{c}{\sqrt{n}(1+\epsilon)}<\frac{1}{Y_{n}}<\frac{c}{\sqrt{n}(1-\epsilon)}
$$

if $0<\epsilon<1$, the first summand in 55 is estimated by

$$
\begin{aligned}
& \mathbb{E}\left(\frac{Y_{m, s}^{(n)}}{Y_{n}} I_{B_{n}^{c}}\right)=\frac{c}{\sqrt{n}}(1+O(\epsilon)) \mathbb{E}\left(Y_{m, s}^{(n)} I_{B_{n}^{c}}\right) \\
& =\frac{c}{\sqrt{n}}(1+O(\epsilon))\left(\mathbb{E}\left(Y_{m, s}^{(n)}\right)-\mathbb{E}\left(Y_{m, s}^{(n)} I_{B_{n}}\right)\right) .
\end{aligned}
$$

Clearly, with probability $1, Y_{m, s}^{(n)} \leq n$. Hence, using 53 , we obtain

$$
\left.\mathbb{E}\left(Y_{m, s}^{(n)} I_{B_{n}}\right)\right)=O\left(n \operatorname{Pr}\left(B_{n}\right)\right)=O\left(n e^{-c_{2} \sqrt{n}}\right)
$$

and 56 becomes

$$
\mathbb{E}\left(\frac{Y_{m, s}^{(n)}}{Y_{n}} I_{B_{n}^{c}}\right)=\frac{c}{\sqrt{n}}(1+O(\epsilon)) \mathbb{E}\left(Y_{m, s}^{(n)}\right)+O\left(n e^{-c_{2} \sqrt{n}}\right) .
$$

The second term in the right hand side of 55 is easily estimated using $(53)$ since it is not greater than $\operatorname{Pr}\left(B_{n}\right)$. Consequently,

$$
\mathbb{P}\left(\mu_{n, 2}=m, \sigma_{n, 2} \leq s\right)=\frac{c}{\sqrt{n}}(1+O(\epsilon)) \mathbb{E}\left(Y_{m, s}^{(n)}\right)+O\left(n e^{-c_{2} \sqrt{n}}\right)
$$

uniformly for any fixed integer $m \geq 1$ and real $s \geq 1$. Hence, our next task is to obtain an estimate for $\mathbb{E}\left(Y_{m, s}^{(n)}\right)$, as $n \rightarrow \infty$, whenever $s=t \sqrt{n} / c, m \geq 1$ is fixed integer and $t \in(0, \infty)$ is also fixed. 
Combining results of (18) and (20) of Lemmas 2 and 3, respectively, and approximating the sum by the corresponding Riemann integral, we get

$$
\begin{aligned}
& \mathbb{E}\left(Y_{m, s}^{(n)}\right)=(1+O(1 / \sqrt{n})) \\
& \times \sum_{1 \leq k \leq c^{-1} \sqrt{n} t}(\exp (-c m k / \sqrt{n})-\exp (-c(m+1) k / \sqrt{n})) \\
& \sim \sqrt{n} \int_{0}^{c^{-1} t}\left(e^{-c m y}-e^{-c(m+1) y}\right) d y .
\end{aligned}
$$

Replacing this expression into (57) and letting first $n \rightarrow \infty$ and then $\epsilon \rightarrow 0$, we obtain

$$
\begin{aligned}
& \mathbb{P}\left(\mu_{n, 2}=m, \frac{c \sigma_{n, 2}}{\sqrt{n}} \leq t\right) \rightarrow c \int_{0}^{c^{-1} t}\left(e^{-c m y}-e^{-c(m+1) y}\right) d y \\
& =\int_{0}^{t} e^{-m y}\left(1-e^{-y}\right) d y,
\end{aligned}
$$

which completes the proof of Theorem 3.2 .

\section{$7 \quad$ Proof of Theorem 3.3}

The proof will be based on an asymptotic analysis of formula $\sqrt{11}$, setting there $s=c^{-1} \sqrt{n} t$ as $n \rightarrow \infty$ (see again (19p) and assuming that $m$ is fixed positive integer. First, we let $\Lambda_{k}(n)$ to denote the set of partitions of $n$ with no part equal to $k$. Also, let $P_{k}(n)=\left|\Lambda_{k}(n)\right|$. In [3] Corteel et al. give a combinatorial proof of the following identity:

$$
\operatorname{Pr}\left(\alpha_{j}^{(n)}=m\right)=\frac{P_{j}(n-m j)}{p(n)}=\frac{p(n-j m)-p(n-j(m+1))}{p(n)} .
$$

Replacing this expression into the right hand side of (11) and applying (20), as in the proof of Theorem 3.2 , we obtain

$$
\begin{aligned}
& \mathbb{P}\left(\mu_{n, 3}=m, \frac{c \sigma_{n, 3}}{\sqrt{n}} \leq t\right) \sim m \sum_{1 \leq j \leq c^{-1} \sqrt{n} t} \frac{j}{\sqrt{n}}\left(e^{-c m j / \sqrt{n}}-e^{c(m+1) j / \sqrt{n}}\right) \frac{1}{\sqrt{n}} \\
& \rightarrow m \int_{0}^{c^{-1} t} y\left(e^{-c m y}-e^{-c(m+1) y}\right) d y=\frac{m}{c^{2}} \int_{0}^{t} y\left(1-e^{-y}\right) e^{-m y} d y .
\end{aligned}
$$

This completes the proof of Theorem 3.3 .

\section{References}

[1] M. Abramovitz and I. A. Stegun , Handbook of Mathemathical Functions with Formulas, Graphs and Mathematical Tables, Dover Publ. Inc., New York, 1965.

[2] G. E. Andrews, The Theory of Partitions, Encyclopedia of Mathematics and its Applications, 2, Addison-Wesley, Reading, MA, 1976. 
[3] S. Corteel, B. Pittel, C. D. Savage And H. S. Wilf, On the multiplicity of parts in a random partition, Random Stuctures Algorithms, 14 (1999) 185-197.

[4] P. ERdös AND J. LeHnER, The distribution of the number of summands in the partition of a positive integer, Duke Math. J., 8 (1941) 335-345.

[5] P. Flajolet and R. Sedgewick, Analytic Combinatorics, Cambridge University Press, Cambridge, 2009.

[6] B. Fristedt , The structure of random partitions of large integers, Trans. Amer. Math. Soc., 337 (1993) 703-735.

[7] B. Granovsky, D. Stark and M. ERlihson, Meinardus theorem on weighted partitions: extensions and a probabilistic proof, Adv. in Appl. Math., 41 (2008) 307-328.

[8] G. Grimmett and D. Stirzaker, Probability and Random Processes, Oxford University Press, Oxford, 2001.

[9] G. H. Hardy and S. Ramanujan, Asymptotic formulae in combinatory analysis, Proc. Lond. Math. Soc., 17 (1918) 75-115.

[10] W. K. Hayman, A generalization of Stirling's formula, J. Reine Angew. Math., 196 (1956) $67-95$.

[11] G. Meinardus, Asymptotische aussagen über partitionen, Math. Z., 59 (1954) 388-398.

[12] L. Mutafchiev, The size of the largest part of random weighted partitions of large integers, Combin. Probab. Comput., 22 (2013) 433-454.

[13] L. Mutafchiev, Sampling part sizes of random integer partitions, Ramanujan J., 37 (2015) $329-343$.

[14] H. Rademacher, On the partition function $p(n)$, Proc. London Math. Soc., 43 (1937) 241254 .

[15] V. N. Sachkov, Combinatorial Methods in Discrete Mathematics, Encyclopedia of Mathematics and its Applications, 55, Cambridge University Press, Cambridge, 1999.

[16] H. WILF, Three problems in combinatorial analysis, J. Combin. Theory Ser. A, 35 (1983) 199-207. 